\title{
DNA Models and Algorithms for NP-complete Problems *
}

\author{
Eric Bach Anne Condon \\ Elton Glaser Celena Tanguay \\ Computer Sciences Department \\ University of Wisconsin \\ 1210 West Dayton Street \\ Madison, WI 57306 USA
}

March 27, 1996

\begin{abstract}
A goal of research on DNA computing is to solve problems that are beyond the capabilities of the fastest silicon-based supercomputers. Adleman and Lipton present exhaustive search algorithms for 3Sat and 3-Coloring, which can only be run on small instances and hence are not practical.

In this paper, we show how improved algorithms can be developed for the 3-Coloring and Independent Set problems. Our algorithms use only the DNA operations proposed by Adleman and Lipton, but combine them in more powerful ways, and use polynomial preprocessing on a standard computer to tailor them to the specific instance to be solved. The main contribution of this paper is a more general model of DNA algorithms than that proposed by Lipton. We show that DNA computation for NPcomplete problems can do more than just exhaustive search. Further research in this direction will help determine whether or not DNA computing is viable for NP-hard problems. A second contribution is the first analysis of errors that arise in generating the solution space for DNA computation.
\end{abstract}

\section{Introduction}

Adleman described how he used standard tools of molecular biology to solve a 7-vertex instance of the Hamiltonian Path problem [1]. A major goal of subsequent research in this area is to understand how DNA computing can be used to solve NP-hard problems.

To address this goal, Lipton [10] and Adleman [2] proposed the following model of DNA computation. A molecular computation proceeds in two phases: solution space generation and computation. The solution space generation phase yields a test tube whose contents (DNA strands) encode strings over

\footnotetext{
* Supported in part by NSF grant numbers CCR 95-10244 and CCR-96-13799 and by matching awards from Thinking Machines Corporation and Digital Systems Corporation. E-mail addresses of the authors are: bach@cs.wisc.edu, condon@cs.wisc.edu,glaserea@cs.wisc.edu, tanguay@cs.wisc.edu. An extended abstract of this work appeared in the proceedings of the 1996 IEEE Complexity Theory Conference.
} 
an alphabet $\Sigma$, whose size can depend on the input. Throughout, we consider a test tube to be a multiset of strings. In each step of the computation phase, an operation is performed on one or more test tubes. The separate operation takes one test tube and a symbol $\sigma \in \Sigma$ and returns two test tubes, one containing the set of strings containing $\sigma$ and the other containing the remaining strings. The merge operation forms the union of two test tubes. The test-if-empty operation tests whether a test tube is empty. Adleman suggests that the test-if-empty operation be used only at the last step of the computation.

To construct the test tube of strings in the solution space generation phase, the following operations are applied to a test tube initially containing empty strings. The append $(\sigma)$ operation appends the symbol $\sigma$ to all strands in a test tube. The split operation partitions a test tube into two such that for each distinct string $x$ in the test tube, if there are $l$ copies of $x$, then there are $l / 2$ copies in each of the two test tubes. (This can be generalized to a split into $k$ test tubes). These operations are used in a very restricted way by Lipton to generate a solution space that represents all possible binary strings of length $n$ and by Adleman [2] to generate a solution space that represents all $3^{n}$ possible colorings of $n$ vertices using 3 colors.

Lipton [10] and Adleman [2] present simple molecular algorithms for 3Sat and 3-Coloring, with solution spaces of size $2^{n}$ and $3^{n}$, respectively. Amos et al. [3] present algorithms for NP-hard problems on a somewhat different DNA computing model. In contrast, the best exact algorithms for standard computing models avoid searching through the whole solution space. Beigel and Eppstein have an $O\left(1.35^{n}\right)$ algorithm for 3-Coloring and Schiermeyer [14] has an $O\left(1.58^{n}\right)$ algorithm for 3Sat. The naive DNA algorithms can't expect to beat these algorithms on any instance size. To see this, suppose we accept Adleman's speculation that a solution space of size $2^{70}$ can be used in a DNA computation. Then, for the 3-coloring problem, the largest possible instance solvable by a DNA computation has 44 vertices. Moreover, if this instance has, say, 400 edges, it would take well over 10 days to perform the DNA computation, assuming optimistically that the average DNA operation takes only 20 minutes (again, from Adleman). In contrast, if we estimate the number of operations for Beigel and Eppstein's algorithm at $1.35^{n}$, that is, we ignore the constant in the running time, then on a (slow!) computer that can perform $10^{6}$ operations per second, a 44-vertex instance of 3-Coloring can be solved in just over half a second, and a 70-vertex instance can be solved in under 22 minutes.

In this paper, we show that if the operations proposed by Adleman and Lipton are combined in more general ways, algorithms for 3-Coloring and Independent Set can be obtained with a much smaller solution space. Our results show that DNA computations can be used for more than simple exhaustive search. Hence, it is premature to reject DNA computing on the basis of the impracticality of exhaustive search. In order to determine if DNA computing is practical, it is important to design and analyze the best possible algorithms we can for this paradigm. Our results represent a first step in this direction. As a bonus, we also get some new and different parallel programming ideas by considering this model. They might be incorporated into future computers that do not use DNA.

The main differences between our model and that of Lipton are in the solution space generation phase. We define our new model carefully in Section 2. In our model, the solution space can be pruned and tailored to the problem instance, avoiding generation of all the combinatorial possibilities. Efficient methods for generating solution spaces other than the set of combinatorial possibilities are interesting in their own right and methods for doing this are already used in combinatorial chemistry [6, 12].

In Section 3, we present our new algorithms. All of our algorithms do a polynomial number of molecular operations. Each algorithm is named by the size of its solution space. For each, we list 
the differences between our model and that of Lipton that allow us to obtain improved bounds on the solution space size.

A $1.89^{n}$ 3-Coloring Algorithm: To obtain this algorithm, we allow test tube contents to be split into weighted subsets and allow splits of already split test tubes in the solution space generation phase. In this way, we can generate the set of strings representing all possible subsets of at most $n / 3$ elements of a set of size $n$. Our algorithm then simply checks if there is an independent subset of vertices of size at most $n / 3$, such that the remaining graph is bipartite. The append operation is used in the computation phase.

A $1.67^{n}$ Independent Set Algorithm: This algorithm uses a polynomial-time preprocessing phase (to be done on a standard computer) that tailors the solution space to the instance to be solved. The computation phase is very simple.

A $2^{n}$ 3-Coloring Algorithm: Although this algorithm has an asymptotically larger solution space than the $1.89^{n}$ algorithm, the computation phase is much simpler. A new feature of this algorithm is the use of the separate operation in a restricted way in the solution space generation phase, in addition to the append and split operations.

A $1.51^{n}$ Independent Set Algorithm: In this algorithm, computation on a standard computer is interleaved with DNA computation throughout. As in the $1.89^{n}$ 3-Coloring algorithm, weighted splits and repeated splits of test tubes are needed.

The second contribution of the paper is analysis of errors due to an imperfect split operation. Lipton's solution space generation model implicitly assumes that in the split operation, every subset of identical strands in the test tube is split perfectly in halves. For example, if a test tube contains 20 strings, say 10 copies each of strings $s_{1}$ and $s_{2}$, then 5 copies of each string appear in each of the two test tubes resulting from the split. In reality however, a split of a test tube is implemented by pouring equal amounts of the contents of a test tube into two test tubes [2]. Even if we assume that the total number of strings in the two test tubes is equal, we can't expect to get a perfect split of each subset of identical strings. Imperfect splits can cause some strings not to be present in the final solution space.

To model this, we replace the split operation with a probabilistic-split operation, in which each possible partition of the test tube into halves is equally likely. This probabilistic model may seem to be an oversimplification. It corresponds, however, to the use of the most probable distribution in statistical mechanics, which usually leads to correct answers. (For example, the Boltzmann distribution is usually derived heuristically in this way.) To ensure that all strings in the solution space are present with high probability, one can place in the initial test tube a number of strings that is many times greater than the size of the desired solution space. To make this precise, for a given error parameter $\epsilon$, we define the redundancy of the solution space generation phase to be an integer such that if the number of strings in the initial test tube is equal to the solution space size times the redundancy, then with probability $1-\epsilon$, the final test tube contains all strings in the desired solution set. This method avoids the need for amplification of strands, which Adleman suggests should be avoided whenever possible.

In Section 4, we analyze the redundancy that is necessary for the solution space generation phases used in our algorithms. To do this, we introduce a new model (graphs with urns) to model the DNA solution space generation process.

We first consider Lipton's model for generating a solution space of size $k^{n}$, and show that a redundancy of $\Theta(n)$ guarantees success with all but exponentially small probability (that is, the probability of success is at least $\left.1-O\left(2^{\Omega(n)}\right)\right)$. The same is true of the solution sets used in our $1.67^{n}$ Independent Set and 
$2^{n}$ 3-Coloring algorithms. The solution space of the remaining algorithms is the set of all binary strings of length $n$ with exactly (or at most) $k$ 1's. We show that a redundancy of $\Theta(n)$ is also sufficient to guarantee success with all but exponentially small probability in this case. A martingale analysis is used to prove these results.

\section{A Model of DNA Computation}

\subsection{Solution Space Generation Phase}

We model the solution space generation algorithm, for a given instance of a problem, using a finite dag called a generator. Associated with each edge of the generator is a test tube. The generator and associated test tubes are defined as follows. The graph has a root vertex with outdegree one and a sink vertex with indegree one. All vertices are reachable from the root vertex and the sink vertex can be reached from all vertices. The vertices of the graph are partitioned into levels, with all edges leaving vertices at level $i$ going to vertices at level $i+1$. Each edge has a positive rational weight (probability), such that the sum of the weights out of a vertex is 1 .

Each vertex is labeled by one of the operations merge, separate, append(symbol), split or no-op. The vertices and multisets associated with the edges are constrained as follows. First, the set associated with the edge leaving the root is a multiset of empty strings. The size of this set is defined to be the size of the solution space generated by the generator.

A split vertex has indegree 1 and arbitrary outdegree. Let the multiset associated with the incoming edge be $M^{\prime}$. The multiset $M$ associated with an outgoing edge of weight $p$ is defined as follows. For each string $s$ in $M^{\prime}$ with multiplicity $m, M$ contains $p m$ copies of $s$. If $p m$ is not an integer, the graph is not a valid generator.

An append(symbol) vertex has indegree 1 and outdegree 1 . Let the multiset assigned to the incoming edge be $M^{\prime}$. Then the multiset associated with the outgoing edge is obtained by appending symbol to the right end of each string of $M^{\prime}$.

A separate vertex has indegree 1 and outdegree 2. One of the outgoing edges is labeled by a symbol. Let $M^{\prime}$ be the multiset associated with the incoming edge. The subset of $M^{\prime}$ that contains symbol is associated with the outgoing edge that is labeled symbol, and the test tube of the remaining strings is associated with the other edge. If an edge is labeled with weight $p$, then the number of strings in the multiset associated with that edge is $p\left|M^{\prime}\right|$; otherwise the graph is not a valid generator.

A merge vertex has outdegree 1 and indegree at least 2 . The multiset associated with the outgoing edge is simply the union of the multisets of all incoming edges. A no-op vertex has indegree 1 and outdegree 1 . The test tube associated with the outgoing edge is simply the test tube associated with the incoming edge. The no-op operation is included simply to make it easy to organize the graph into levels.

The multiset of strings associated with the edge entering the sink vertex is the solution set (possibly multiset) generated by the generator. The size of this set is the same as the size of the multiset associated with the edge leaving the root.

Examples. Lipton proposed a generator (in the case $k=2$ ) to generate a solution set representing all $k^{n}$ strings over an alphabet of size $k$. The strings in the solution space are of the form $b_{1} b_{2} \ldots b_{n}$ 
where $b_{i} \in\left\{1_{i}, 2_{i}, \ldots, k_{i}\right\}$. The generator consists of a sequence of $n$ split nodes of outdegree $k$, where the $j$ th edge from the $i$ th split node leads to an append $\left(j_{i}\right)$ node, followed by a $k$-way merge. We call this a $k^{n}$-generator.

In Figure 3, we present a generator that generates a solution set representing the set of all binary strings of length $n$ with exactly $k$ 1's. We call this a $\left(\begin{array}{c}n \\ k\end{array}\right)$-generator.

\section{$2.2 \quad$ A DNA Model for Languages}

Let $L$ be a problem in NP. A $D N A$ algorithm for $L$ is a polynomial time algorithm (for a standard computer) with the following properties. Given an instance $x$ of $L$, it outputs a (valid) generator for $x$ and a specification of a DNA computation phase. The DNA computation phase may be specified by a dag similar to a generator, except that there are no weights associated with the edges, vertices need not be organized into levels, there are no vertices labeled with the operations split or no-op, and the last ( $\sin k)$ vertex is labeled with test-if-empty. The test tube labeling the edge from the root is the output of the generator; test tubes labeling the other edges are the result of the operations on the vertices. The test tube labeling the edge into the sink is not empty if and only if the instance is in $L$.

There are several resources of a DNA algorithm that are important to measure, as a function of input size. The primary ones that we consider are: (i) the solution space size, (ii) the total length of the computation, measured as the length of the path from root to sink in the generator, plus the maximum length of a path from root to sink in the dag specifying the DNA computation, (iii) the number of operations performed during the solution space generation phase and the computation phase, and (iv) the number of test tubes needed. Another resource that we will introduce later is the redundancy needed due to the probabilistic nature of the low-level split operation.

Algorithms for 3Sat, 3-Coloring and other problems can be found in $[2,10]$. The 3SAT algorithm has a solution space of size $2^{n}$ and requires $\Theta(n+m)$ operations, where $n$ is the number of variables and $m$ is the number of clauses. The 3 -Coloring algorithm has a solution space of size $3^{n}$ and requires $\Theta(n+m)$ operations, where $n$ is the number of nodes and $m$ is the number of edges. An algorithm for the Circuit Sat problem can be found in [5]. This problem is to determine if an $n$-input circuit (with "and", "or" and "not" gates and one designated output gate) has an input that sets the output to 1. Their algorithm has a solution space of size $2^{n}$ on an instance circuit with $n$ inputs. The output of the solution space generator is simply the test tube consisting of all $2^{n}$ possible inputs to the circuit. Briefly, in the DNA computation, the gates of the circuit are processed in topological order. When processing gate $g$, inputs for which $g$ evaluates to 1 are separated from those for which $g$ evaluates to 0 . The appropriate value of $g$ is appended to each input (in parallel for each of the two possible values of $g$ ), and the two test tubes are merged. In this way, the value of $g$ is available when processing other gates that have the output of $g$ as input. In this algorithm, the size of each element of the solution space is initially of length $n$ but increases to length $n+m$, where $m$ is the number of gates in the circuit.

More generally, if a test tube contains some subset of the $2^{n}$ possible inputs to a circuit, the same algorithm can be used to determine if any element of this subset sets the output of the circuit to 1 . Furthermore, if the circuit has several outputs, then the same algorithm transforms the initial test tube into a test tube in which each possible input to the circuit has appended to it the value of every output gate. 


\begin{tabular}{|l|l|l|l|l|l|}
\hline Problem & $\begin{array}{l}\text { Solution } \\
\text { Space Size }\end{array}$ & Redundancy & $\begin{array}{l}\text { Length of } \\
\text { Computation }\end{array}$ & $\begin{array}{l}\text { Number of } \\
\text { Operations }\end{array}$ & $\begin{array}{l}\text { Number of } \\
\text { Test Tubes }\end{array}$ \\
\hline \hline 3-Coloring & $1.89^{n}$ & $\Theta(n)$ & $\Theta\left(n^{2}+m\right)$ & $\Theta\left(n^{2}+m^{2}\right)$ & $\Theta(n)$ \\
& $2^{n}$ & $\Theta(n)$ & $\Theta(m)$ & $\Theta(m)$ & $\Theta(1)$ \\
\hline Ind Set & $1.67^{n}$ & $\Theta(n)$ & $\Theta(m)$ & $\Theta(m)$ & $\Theta(n)$ \\
& $1.51^{n}$ & $\Theta(n)$ & $O\left(n^{2} m^{2}\right)$ & $O\left(n^{2} m^{2}\right)$ & $\Theta\left(n^{2}\right)$ \\
\hline
\end{tabular}

Table 1: Asymptotic bounds for resources required by the four DNA algorithms.

\section{New Algorithms}

We present four algorithms that exploit our DNA computing model in different ways. Each algorithm is described by the size of its solution space, since this is the most expensive resource and thus the one that is most important to minimize. In what follows, we assume that the input to each algorithm is a connected, undirected graph $G=(V, E)$, where $|V|=n$ and $|E|=m$. We summarize the resources used by each algorithm in Table 1.

A $1.89^{n}$ 3-Coloring Algorithm. The generator produces a test tube $T$ of strings that represent all subsets of $V$ of size $\leq \frac{n}{3}$. Each string in the solution space is of the form $b_{1} b_{2} \ldots b_{n}$ where $b_{i} \in\left\{0_{i}, 1_{i}\right\}$; this string represents the subset of vertices $i$ of $V$ for which $b_{i}=1_{i}$. Roughly, this can be done by a generator that is formed by merging the results of the generators from Figure 3 that generate strings with exactly $1,2, \ldots, k 1$ 's. The generator is of size $O\left(n^{3}\right)$ and has length $n$. T contains $\sum_{j=1}^{\frac{n}{3}}\left(\begin{array}{c}n \\ j\end{array}\right)$ elements (assuming $n$ is a multiple of 3 for simplicity). Applying Stirling's formula, we have that $\sum_{j=1}^{\frac{n}{3}}\left(\begin{array}{c}n \\ j\end{array}\right) \leq n\left(\begin{array}{c}n \\ 3\end{array}\right)=n\left(\frac{9}{4 \pi n}\right)^{1 / 2}\left(\frac{n}{n / 3}\right)^{n / 3}\left(\frac{n}{2 n / 3}\right)^{2 n / 3}(1+O(1 / n)) \leq\left(\frac{9 n}{4 \pi}\right)^{1 / 2} 1.8899^{n}(1+O(1 / n)) \leq 1.89^{n}$ for sufficiently large $n$. (In fact, $\sum_{j=1}^{\frac{n}{3}}\left(\begin{array}{c}n \\ j\end{array}\right) \leq 1.89^{n}$ for all integer multiples of 3 ).

In the computation phase, the elements of $T$ that are not independent subsets of $V$ are first discarded. To do this, for every edge $e$ of $G$, those elements of the solution space containing both endpoints of $e$ are separated from those containing one or none of $e$ 's endpoints, and discarded. The elements remaining in $T$ are independent subsets of $V$ of size at most $n / 3$. Then, for each remaining element $s$ of $T$, the algorithm tests if the subgraph of $G$ induced by the set $V-s$ is bipartite. This can be done in $O\left(n^{2}+m\right)$ time (details omitted).

A $1.67^{n}$ Independent Set Algorithm. Briefly, this algorithm uses a preprocessing phase to identify small, well-connected components in $G$. The solution space is then constructed to represent the set of subsets of the vertices of the graph that contain no adjacent pair of vertices from the same component.

The generator first selects a maximal set $S$ of vertex-disjoint subgraphs from $G$, with exactly 4 vertices and at least 4 edges. These are called 4-components. A case enumeration of the possibilities shows that each such 4-component contains at most 7 distinct independent sets out of the 16 possibilities. From the remaining vertices of $G$, a maximal set of vertex-disjoint subgraphs that are connected and have exactly 5 vertices are selected and these 5 -components are added to $S$. Any such 5 -component contains at most 13 independent sets. Let $V^{\prime}$ be the set of vertices in the components of $S$. Note that there may 
still be vertices in $G-S$.

The solution space consists of strings of the form $b_{1} b_{2} \ldots b_{\left|V^{\prime}\right|}$, where $b_{i} \in\left\{0_{i}, 1_{i}\right\}$. This string represents the subset of vertices $i \in V^{\prime}$ for which $b_{i}=1_{i}$. Moreover, no subset (string) in the solution space contains a pair of vertices that are neighbors in a 4-component or a 5-component of $S$. Initially, the solution space consists of a multiset $T$ of empty sets. For each 4-component in turn, a 7-way split is performed on $T$. A different combination of $41_{i}$ 's and $0_{i}$ 's are appended to each of the 7 resulting test tubes, corresponding to the 7 different independent sets contained in the 4 -component. Then, the 7 sets are merged back together to reform $T$. 5-components are handled in an analogous manner, except that the solution space is split 13 ways and 5 vertices are appended to each of the 13 test tubes. It is not hard to see that the worst-case size of the solution set occurs when all components in $S$ are 5 -components, and that the number of 5 -components is at most $n / 5$. Therefore, the worst-case size of the solution space is at most $13^{n / 5}=1.67^{n}$.

The computation phase proceeds as follows. First, for every edge $e$ connecting vertices in two different components of $S$, those elements of the solution space containing both endpoints are separated from those containing one or none of $e$ 's endpoints, and discarded. The elements remaining in $T$ are exactly the independent subsets of $V^{\prime}$.

Next, the vertices in $G-S$ are handled. The goal is to add as many of these remaining vertices as possible to each element of the solution space while ensuring that each element remains an independent set. A vertex $i$ can be "added" to the solution set by first separating out all independent sets which contain a neighbor of $i$, and appending $0_{i}$ to these strings. Then $1_{i}$ is appended to the remaining strings. This ensures that the elements of $T$ remain independent subsets of $G$, but the vertices of $G-S$ must be "added" in an order that guarantees that the maximum independent set will be in the solution space. This can be done by exploiting the fact that every connected component in $G-S$ is either a triangle or a tree.

Any triangle in $G-S$ is not connected to any other vertex in $G-S$, because otherwise that 4 component could have been added to $S$. Since at most one of the 3 vertices in a triangle can be in an independent set, they can be added in an arbitrary order. For each tree in $G-S$, a vertex is chosen arbitrarily to be the root of the tree. Then, vertices are added in postfix order, which ensures that the children of a vertex are added before that vertex itself. This order ensures that the maximum number of vertices of the tree are being added.

Finally, the algorithm determines whether an element of the resulting test tube $T$ is of cardinality at least $k$. To do this, $T$ is partitioned into test tubes $T_{i}, 0 \leq i \leq k$, such that for $i<k$, each $T_{i}$ contains all solutions of size $i$ and $T_{k}$ is contains all solutions of size at least $k$. Initially, $T_{0}=T$ and the remaining test tubes are empty. Then, all solutions containing vertex 1 are separated from $T_{0}$ and merged with $T_{1}$. More generally, for every vertex $i$, for every test tube $T_{j}$, all solutions containing $t_{i}$ are separated from $T_{j}$ and merged with $T_{j+1}$. After this is done for all vertices $i$, all elements of the solution space with at least $k$ vertices are in $T_{k}$. A test-if-empty operation performed on $T_{k}$ determines whether $G$ has an independent set of size at least $k$.

A $2^{n}$ 3-Coloring Algorithm. This algorithm is the first to use the separate operation in the solution space generation phase. The DNA algorithm first selects a rooted spanning tree of $G$, and fixes the color of the root (vertex 1). The solution space consists of strings of the form $c_{1} c_{2} \ldots c_{n}$ where each $c_{i} \in\left\{b_{i}, r_{i}, g_{i}\right\}$, representing the fact that vertex $i$ can have color blue, red or green. Moreover, in any string, $c_{1}$ is the fixed root color, and if $i$ is $j$ 's parent in the tree, then $j$ cannot have the same color 
as $i$. This solution space is constructed as follows. Initially, the solution space consists of a test tube $T$ of empty strings. The vertices of the spanning tree are considered in prefix order. When considering vertex $i, T$ is separated into 3 different tubes, say $B, R$ and $G$, based on the color of $i$ 's parent, $j$, in the tree. Then, test tube $B$ is split in $2, r_{i}$ is appended to one and $g_{i}$ to the other. Test tubes $R$ and $G$ are handled similarly. Finally, all 6 test tubes are merged to reform $T$.

The computation phase then simply selects out those solutions which are not valid colorings by examining each edge of $E$ that is not in the spanning tree in turn, and discarding strings in which both endpoints are the same color.

A $1.51^{n}$ Independent Set Algorithm. Briefly, this algorithm tries to find two disjoint independent sets of size $n / 7$ in $G$, which induce a bipartite subgraph $B$. After generating a solution space of independent sets of $G-B$, the vertices of $B$ are added later, without increasing the size of the solution space. Although we obtain an improved solution space size, the required computation includes simulation of a circuit for bipartite matching. We assume that the problem is to find an independent set of size $k \geq 2 n / 7$; otherwise, we can solve the problem with an abbreviated version of our algorithm which has an even smaller solution space.

The DNA computation first determines if there is an independent set of size $n / 7$. The generator of Figure 3 is used to generate all subsets of $V$ of size $n / 7$. The solution space is of size at most $1.51^{n}$. Then, the computation phase discards all those subsets that are not independent (as in previous algorithms), and a test-if-empty operation is performed on the test tube of remaining strings. If there is an independent set $I$ of size $n / 7$ in this test tube, our algorithm needs to compute $I$ in order to proceed. This could be done by extending our model to include a "decode" operation that returns the value of a string in a test tube. We can still compute $I$ without this operation, but in a very brute force fashion that requires the solution space generation and computation phases to be repeated $n / 7$ times, with one vertex of $I$ being computed each time. In the same way, the algorithm tries to find another independent set $J$ of size $n / 7$ in the graph $G$ with the subgraph induced by $I$ removed. The solution space for this computation is of size at most $1.47^{n}$. If no independent set is found in either of the first two steps, then we know that there are no independent sets of size $k>2 n / 7$. Otherwise, the algorithm continues as follows.

Next, a solution space containing all independent sets of $G-B$ is computed. Let $B$ be the bipartite subgraph of $G$ induced by $I \cup J$. Let $P$ be a maximal set of disjoint pairs of adjacent vertices from $G-B$ and let $S$ be the independent set of vertices of $G-B$ that are not in $P$. We can bound the size of $S$ from above by $n / 7$, by simply swapping $I$ and $S$ if $S$ exceeds $n / 7$ vertices. The solution space consists of subsets of $V-I-J$ that contain no pair of $P$. Note that each pair of vertices in $P$ contains 3 independent sets out of the four possibilities. Hence, the size of this solution space is $2^{|S|} 3^{|P|}$. Obviously, the worst-case solution space results when $|S|$ is maximized at $n / 7$. With $n / 7$ of $G$ 's vertices in $S$, at least $n / 7$ in $I$, and $n / 7$ in $J$, at most $4 n / 7$ vertices ( $2 n / 7$ pairs) remain in $P$. Hence, the worst-case solution space size is $3^{2 n / 7} \times 2^{n / 7}=1.51^{n}$. Let $T$ be the resulting test tube. All elements of $T$ that are not independent sets are discarded.

Finally, the vertices of $B$ are added to the elements of $T$ in the following way. For every vertex $i$ in $B$, append $1_{i}$ to those elements of $T$ which do not contain a neighbor of $i$ from the set $V-I-J$, and $0_{i}$ to the remaining sets. The resulting test tube $T$ contains strings that represent sets of vertices that induce bipartite subgraphs of $G$. Moreover, each maximum independent set of $G$ is a subset of at least one of these sets. The algorithm must now find the maximum independent sets of the bipartite graphs of $T$. 
We observe that this problem can be solved with a circuit of size $O\left(n^{2} m^{2}\right)$ (using bipartite matching; see [9]). The circuit has $n$ inputs representing the presence or absence of each vertex of $G$ in a bipartite graph, and $n$ outputs which indicate the maximum independent set. The circuit first finds a maximal matching $M$ in the bipartite graph. Then, for each of the $O(n)$ free nodes (i.e. nodes not incident to an edge in $M$ ), the circuit performs a breadth-first search along alternating paths beginning at the free node. The search is simulated by repeatedly expanding sets of edges along disjoint alternating paths. After all eligible edges have been covered, the circuit searches through the sets of edges to find an edge which completes an augmenting path (an alternating path with a free node at each end). If an alternating path is found, it can be "inverted" to increase the size of the current matching by one. Once these three steps (each requiring $O\left(\mathrm{~nm}^{2}\right)$ gates) have been performed on every free node, the resulting matching is maximum. A maximum independent set can then be determined from this matching by selecting any remaining free nodes, as well as one node from each edge in the matching. One node from every edge is guaranteed to be in a maximum independent set; otherwise, we would contradict the assumption that the matching is optimal. A DNA computation can simulate this circuit, resulting in the $n$ outputs being appended to each element of $T$.

All that remains is to isolate those outputs of size at least $k$. This process is identical to the final step of the $1.67^{n}$ independent set algorithm.

\section{Probabilistic Implementation of Split Operation}

Suppose that in a generator, the split vertex is replaced by the following probabilistic-split vertex. Suppose that the edges leaving this vertex are weighted $p_{1}, p_{2}, \ldots, p_{k}$. Then, the operation, applied to a test tube with $N$ strings, produces a partition of the test tube into $k$ test tubes containing $p_{1} N, p_{2} N, \ldots, p_{k} N$, strings, respectively. Moreover, each possible such partition is equally likely. In this section, for several graph types, we describe the redundancy that is then needed in the solution space.

We first consider the $n$-vertex $k^{n}$-generator of Lipton. Let $p=1 / k$ and let $M=1 / p^{n}$. Our goal is to find bounds on the number $N$ of strings needed in the initial test tube in order that all $M$ distinct strings are generated with high probability. The distribution of strings produced by probabilisticsplit equals the distribution produced by the following process. Suppose that instead of generating all strings in parallel, one string at a time is generated. The generation of a string is called a trial. The string generated at each trial is determined by a path chosen randomly in the graph in the following way. Associated with each split vertex is an urn, which initially has $N$ balls of $k$ different colors, with exactly $p N$ of each color. At each trial, a ball is removed from every urn. The color of the ball determines which edge is in the path.

In the first trial, each of the possible $M$ strings are equally likely. If the same were true in every trial, that is, if each of the $M$ strings is equally likely, then solutions to the coupon collector's problem show that $N=\Theta(M \log M)$ trials are necessary and sufficient, in order that all $M$ distinct strings are generated with high probability. We note that when the output of the $k^{n}$ generator is used to find an optimal solution for an NP-hard problem, it is not necessary that all members of the solution space are present in the generated solution space, but only that the optimal solution is present. However, if again each of the $M=k^{n}$ strings is equally likely in every trial, then $\Theta(M \log M)$ trials are still needed to ensure that one fixed string out of the $M$ possibilities is generated with probability at least $1-2^{-\Theta(n)}$. In our urn model, the probability of generating a particular string in a given trial depends on the strings 
generated in previous trials. We show in Theorem 1 that it is still the case that $N=\Theta(M \log M)$ trials are needed to generate all strings with high probability. First, we introduce some notation and state a key lemma, which will be proved in Section 4.1.

Let red be one of the colors used in the urns. Let $X_{t}$ be the number of red balls after $t$ samples from the urn, so that $X_{0}=p N, X_{1}=p N$ with probability $1-p$ or $p N-1$ with probability $p$, and so on. The key lemma is:

Lemma 1 For any $\epsilon>0$, with probability at least $1-2 \exp \left(-p^{2}(\log N) / 16\right)$,

$$
\left|X_{t}-E\left[X_{t}\right]\right|<p(N \log N)^{1 / 2}, 1 \leq t \leq N / 2 .
$$

This lemma shows that in the first $N / 2$ trials, the choice of ball from an urn is relatively unbiased, with high probability. As a result of the lemma, each of the first $N / 2$ strings is chosen in an almost unbiased manner, and so $N$ is as predicted by the coupon collector's problem. This is made precise in the following theorem, whose proof is deferred to the next section.

Theorem 1 To obtain exponentially small error, a redundancy of $\Theta(n)$ is sufficient for the $k^{n}$-generator. More precisely, suppose that $n \leq(3 / 8)(N / \log N)^{1 / 2}$ and that $N \geq 8 c M \log M$. Then all $M=k^{n}$ strings are generated in the $k^{n}$-generator with probability at least

$$
1-\frac{2 n}{p} \exp \left(-p^{2}(\log N) / 16\right)-M^{1-c}
$$

The generator for the $1.67^{n}$ Independent-Set algorithm is very similar to a $k^{n}$-generator, except the outdegrees of the split vertices may not all be equal. A bound is obtained simply by letting $k_{\max }=13$ be the maximum outdegree of a vertex, $p_{\min }=1 / k_{\max }$ and using these values in the proof of Theorem 1. Thus, a redundancy of $\Theta(n)$ is necessary and sufficient to guarantee that the desired solution space is generated with all but exponentially small probability.

Theorem 2 Suppose that $2 n \leq(N / \log N)^{1 / 2}$ and that $N \geq 8 c M \log M$. Then with probability at least

$$
1-\frac{2 n}{p_{\min }} \exp \left(-p_{\text {min }}^{2}(\log N) / 16\right)-M^{1-c},
$$

all $M=1.67^{n}$ strings are generated by the generator for the $1.67^{n}$ Independent Set algorithm.

In the $2^{n} 3$-Coloring algorithm, the split operations are applied to test tubes with $N / 3$ strings, rather than $N$ strings. Moreover, the number of split vertices for an $n$-vertex graph is $3 n$. The same analysis as in Theorem 1 can be applied with these changes, and the fact that $p=1 / 3$, to see that the redundancy is again $\Theta(n)$.

Theorem 3 Suppose that $2 n \leq((N / 3) / \log (N / 3))^{1 / 2}$ and that $N \geq 8 c M \log M$. Then with probability at least

$$
1-6 \exp (-9(\log (N / 3)) / 16)-M^{1-c}
$$

all $M=2^{n}$ strings are generated in the generator for the $2^{n}$-Coloring algorithm.

Finally, we have a bound of $\Theta(n)$ on the redundancy for the $\left(\begin{array}{c}n \\ n / k\end{array}\right)$ generator that is used in our $1.89^{n}$ 3-Coloring and $1.51^{n}$ Independent Set algorithms.

Theorem 4 Let $k>1$ be an integer. A redundancy of $R=\Theta(n)$ is sufficient for the $\left(\begin{array}{c}n \\ n / k\end{array}\right)$-generator, to obtain exponentially small error. More precisely, suppose that $N(=R M) \geq 8 c M \log M$. Then for sufficiently large $n$, all $M=\left(\begin{array}{c}n \\ n / k\end{array}\right)$ strings are generated with probability at least $1-2^{-\Omega(n)}-M^{1-c}$. 


\subsection{Proofs}

In this section, we give the proofs of the main results stated above. First, we check that the urn models give the same distribution of output strings as the probabilistic split operation. This is an example of the "principle of deferred decisions," which has been emphasized by Knuth [7]. We state it as a lemma:

Lemma 2 Let $n_{1}, \ldots, n_{k}$ be nonnegative integers summing to $n$. We draw from an urn initially containing $n_{i}$ balls of color $i, i=1, \ldots, k$, and assign the integer $j$ to the $j$-th color obtained in this fashion. This produces a random partition of $\{1, \ldots, n\}$ into $k$ classes, subject to the constraint that the $i$-th class have $n_{i}$ elements.

Proof: Considering for the moment the balls to be distinguishable, there are $n$ ! ways to draw from the urn. The number of such draws that lead to a particular partition is the multinomial coefficient

$$
\left(\begin{array}{c}
n \\
n_{1} \cdots n_{k}
\end{array}\right),
$$

and this number does not depend on the partition. Hence the partitions are uniformly distributed, subject to the constraint.

We now connect this to our DNA models. Suppose, for example, we generate $M$ strings of length $n$ using an $n$-vertex $k^{n}$ generator. For each $j$, the $j$-th symbols of these strings induce a random partition of $\{1, \ldots, M\}$ into $k$ equinumerous classes. So it does not matter whether we think of these symbols as being chosen all at once (by distributing $M / k$ copies of each symbol among the strings), or one at a time (by using an urn to choose the symbols). We note also that the various symbols are independent, in the sense that knowledge of the $i$-th symbol (for any subset of the $M$ strings) does not give us any information about the $j$-th.

Our next goal is to prove Lemma 1. Because of the independence, we can focus attention on one of the urns. Let $X_{i}$ denote the number of red balls after $i$ samples have been chosen from the urn. First, we note that

$$
\begin{aligned}
E\left[X_{i+1} \mid X_{i}, X_{i-1}, \ldots, X_{0}\right] & =X_{i}-\operatorname{Pr}[\text { we draw a red ball }] \\
& =X_{i}-X_{i} /(N-i) \\
& =X_{i}\left(1-\frac{1}{N-i}\right)
\end{aligned}
$$

The right hand expression is a multiple of $X_{i}$ (albeit one depending on $i$ ), which suggests we should try to find a martingale. A sequence of random variables with finite expectation $X_{0}, X_{1}, \ldots$ is said to be a martingale sequence if for all $i>0, E\left[X_{i} \mid X_{0}, \ldots, X_{i-1}\right]=X_{i-1}$.

We can do this by normalizing the variables so as to cancel out the effect of the multiplier. Let $a_{i}=1-1 /(N-i)$, and define a new variable

$$
Z_{i}:=\frac{X_{i}}{a_{i-1} \ldots a_{1} a_{0}} .
$$

Then we have

$$
E\left[Z_{i+1} \mid Z_{i}, Z_{i-1}, \ldots, Z_{0}\right]=\frac{X_{i} a_{i}}{a_{i} \ldots a_{1} a_{0}}=Z_{i}
$$

so $\left\{Z_{i}\right\}$ is a martingale. We note that

$$
a_{i-1} \ldots a_{1} a_{0}=\frac{N-i}{N}
$$


We will employ a standard result of martingale theory, which states that a bounded-difference martingale acts like a sum of independent random variables, in the sense that it is unlikely to wander far from its mean.

Theorem 5 (Hoeffding/Azuma Inequality) Let $Z_{0}, Z_{1}, \ldots$ be a martingale such that for each $i$,

$$
\left|Z_{i}-Z_{i-1}\right| \leq c_{i}
$$

Then, for all $t \geq 0$ and any $\lambda \geq 0$,

$$
\operatorname{Pr}\left[\exists i \leq t\left|Z_{i}-Z_{0}\right| \geq \lambda\right] \leq 2 \exp \left(-\frac{\lambda^{2}}{2 \sum_{i=1}^{t} c_{i}^{2}}\right) .
$$

Proof: See Williams [16], p. 237.

This is a bit stronger than the usual version appearing in textbooks (e.g. Grimmett and Stirzaker [8]), in that it bounds the probability that any sample, not just the last one, deviates far from the mean.

To use this result we must bound the differences of $Z_{i}$.

Lemma 3 If $1 \leq i \leq N / 2$, then $\left|Z_{i}-Z_{i-1}\right| \leq 4$.

Proof: We write $Z_{i}=\alpha_{i} X_{i}$, so that

$$
\alpha_{i}=\frac{N}{N-i}
$$

Note that

$$
Z_{i}-Z_{i-1}=\alpha_{i}\left(X_{i}-X_{i-1}\right)+\left(\alpha_{i}-\alpha_{i-1}\right) X_{i-1} .
$$

In the range $1 \leq i<N / 2$, we have $1 \leq \alpha_{i} \leq 2$. Also, some algebra shows that

$$
\alpha_{i}-\alpha_{i-1}=\frac{\alpha_{i} \alpha_{i-1}}{N}
$$

The result follows easily, since $X_{i-1} \leq N$ and $X_{i}-X_{i-1}$ can only be 0 or -1 .

We can now prove Lemma 1 and Theorem 1, which we restate for the convenience of the reader.

Lemma 1 For any $\epsilon>0$, with probability at least $1-2 \exp \left(-p^{2}(\log N) / 16\right)$,

$$
\left|X_{t}-E\left[X_{t}\right]\right|<p(N \log N)^{1 / 2}, 1 \leq t \leq N / 2 .
$$

Proof: Assume that $1 \leq t \leq N / 2$. By Theorem 5 and Lemma 3 , we have

$$
\begin{aligned}
\operatorname{Pr}\left[\left|Z_{t}-Z_{0}\right| \geq \lambda\right] & \leq 2 \exp \left(-\frac{\lambda^{2}}{2 \sum_{i=1}^{t} c_{i}^{2}}\right) \\
& \leq 2 \exp \left(-\frac{\lambda^{2}}{32 t}\right) \\
& \leq 2 \exp \left(-\frac{\lambda^{2}}{16 N}\right) .
\end{aligned}
$$


Also, if $\left|Z_{t}-Z_{0}\right|<\lambda$, then

$$
\begin{aligned}
\left|X_{t}-E\left[X_{t}\right]\right| & =\frac{1}{\alpha_{t}}\left|Z_{t}-E\left[Z_{t}\right]\right| \\
& =\frac{N-t}{N}\left|Z_{t}-Z_{0}\right| \\
& <\lambda .
\end{aligned}
$$

Therefore,

$$
\begin{aligned}
& \operatorname{Pr}\left[\text { for all } t \leq N / 2,\left|X_{t}-E\left[X_{t}\right]\right|<\lambda\right] \\
\geq & \operatorname{Pr}\left[\text { for all } t \leq N / 2,\left|Z_{t}-Z_{0}\right|<\lambda\right] \\
\geq & 1-2 \exp \left(-\frac{\lambda^{2}}{16 N}\right)
\end{aligned}
$$

Choose $\lambda=p(N \log N)^{1 / 2}$. Then the last expression is $1-N \exp \left(-p^{2}(\log N) / 16\right)$.

Theorem 1 To obtain exponentially small error, a redundancy of $\Theta(n)$ is sufficient for the $k^{n}$ generator. More precisely, suppose that $n \leq(3 / 8)(N / \log N)^{1 / 2}$ and that $N \geq 8 c M \log M$. Then all $M=k^{n}$ strings are generated in the $k^{n}$-generator with probability at least

$$
1-\frac{2 n}{p} \exp \left(-p^{2}(\log N) / 16\right)-M^{1-c}
$$

Proof: Call an urn good for the color red if the fraction of red balls it contains is

$$
\geq p\left(1-\frac{2 \sqrt{\log N}}{N^{1 / 2}}\right) .
$$

Recall that $p=1 / k$ and that the number of red balls in the urn associated with each node initially (i.e. before any strings are generated) is $p N$. Now fix a string $s$, and let the edges on the path that generates it correspond to red balls in the urns. If all of these urns are good, then the probability of generating $s$ is at least

$$
\frac{1}{M}\left(1-\frac{2 \sqrt{\log N}}{N^{1 / 2}}\right)^{n} \geq \frac{1}{4 M}
$$

provided that $n \leq(3 / 8)(N / \log N)^{1 / 2}$.

We now introduce another urn model $U^{\prime}$. This acts just like the original model $U$, except that if an urn becomes bad for some color, it is immediately refilled with its original contents. To keep things straight, we will use $\operatorname{Pr}$ for probabilities under the original urn model, and $\mathrm{Pr}^{\prime}$ for probabilities under $U^{\prime}$.

Let $E_{i}$ be the event that $U^{\prime}$ fails to output the string $s$ during the first $i$ trials. We will show that

$$
\operatorname{Pr}^{\prime}\left[E_{i}\right] \leq\left(1-\frac{1}{4 M}\right)^{i}
$$

Why is this? Intuitively, we have doctored the probabilities so that the chance of getting any particular string is $\geq 1 /(4 M)$, independent of the previous history. 
More formally, we can make the following argument. Since $E_{i-1}$ is determined by the history $x$ of the first $i-1$ trials, we have

$$
\begin{aligned}
\operatorname{Pr}^{\prime}\left[E_{i} \mid E_{i-1}\right] & =\sum_{x \in E_{i-1}} \operatorname{Pr}^{\prime}\left[E_{i} \mid x\right] \frac{\operatorname{Pr}^{\prime}[x]}{\operatorname{Pr}^{\prime}\left[E_{i-1}\right]} \\
& \leq \sum_{x \in E_{i-1}}(1-1 / 4 M) \frac{\operatorname{Pr}^{\prime}[x]}{\operatorname{Pr}^{\prime}\left[E_{i-1}\right]} \\
& =(1-1 / 4 M) .
\end{aligned}
$$

Then since $E_{i} \subset E_{i-1} \subset \ldots \subset E_{1}$,

$$
\operatorname{Pr}^{\prime}\left[E_{i}\right]=\operatorname{Pr}^{\prime}\left[E_{i} \cap \ldots \cap E_{1}\right]=\operatorname{Pr}^{\prime}\left[E_{i} \mid E_{i-1}\right] \cdots \operatorname{Pr}^{\prime}\left[E_{2} \mid E_{1}\right] \operatorname{Pr}^{\prime}\left[E_{1}\right] \leq(1-1 /(4 M))^{i}
$$

Let $G_{i}$ (for "good") be the event that all urns are good (for all colors) before each of the first $i$ trials. Let $B_{i}$ be the complement of $G_{i}$. As long as all urns are good, there is no difference between $U$ and $U^{\prime}$, so we have

$$
\begin{aligned}
\operatorname{Pr}\left[E_{i}\right] & \leq \operatorname{Pr}\left[E_{i} \cap G_{i}\right]+\operatorname{Pr}\left[B_{i}\right] \\
& =\operatorname{Pr}^{\prime}\left[E_{i} \cap G_{i}\right]+\operatorname{Pr}\left[B_{i}\right] \\
& \leq \operatorname{Pr}^{\prime}\left[E_{i}\right]+\operatorname{Pr}\left[B_{i}\right] \\
& \leq(1-1 /(4 M))^{i}+\operatorname{Pr}\left[B_{i}\right]
\end{aligned}
$$

Let us now write $E_{i}(s)$ for the event that the string $s$ fails to appear in trials $1, \ldots, i$. We have

$$
\begin{aligned}
\operatorname{Pr}\left[\exists s E_{i}(s)\right] & \leq \operatorname{Pr}\left[\exists s E_{i}(s) \cap G_{i}\right]+\operatorname{Pr}\left[B_{i}\right] \\
& \leq M(1-1 / 4 M)^{i}+\operatorname{Pr}\left[B_{i}\right],
\end{aligned}
$$

obtaining the last inequality as before.

Now take $i=N / 2$. At the $t$-th trial, we have

$$
E\left[X_{t}\right]=\frac{N-t}{N} X_{0}=p(N-t) .
$$

Using this in Lemma 1, we find (using $t \leq N / 2$ ) that

$$
\operatorname{Pr}\left[B_{N / 2}\right] \leq \frac{2 n}{p} \exp \left(-p^{2}(\log N) / 16\right),
$$

by summing over all colors and all urns. If $N \geq 8 c M \log M$, we also have the estimate

$$
M(1-1 / 4 M)^{N / 2} \leq M \exp (-N / 8 M) \leq M^{1-c} .
$$

Combining these results, we obtain Theorem 1.

Theorems 2 and 3 are proved similarly, and we leave the proofs to the reader. 
We will now study the $\left(\begin{array}{c}n \\ n / k\end{array}\right)$ generators in detail. These generators are based on the idea that the number of monotonic paths linking the opposite corners of an $\ell \times m$ rectangular lattice is given by the binomial coefficient $\left(\begin{array}{c}\ell+m \\ m\end{array}\right)$.

It will be helpful to think of these generators as having numbers written on their edges, in the following way. Suppose some vertex has an urn containing $r$ red balls, the drawing of which directs us along the edge $e$. Then we write $r$ on that edge. For example, the initial state of the $\left(\begin{array}{l}6 \\ 3\end{array}\right)$ generator is given by Figure 1 .

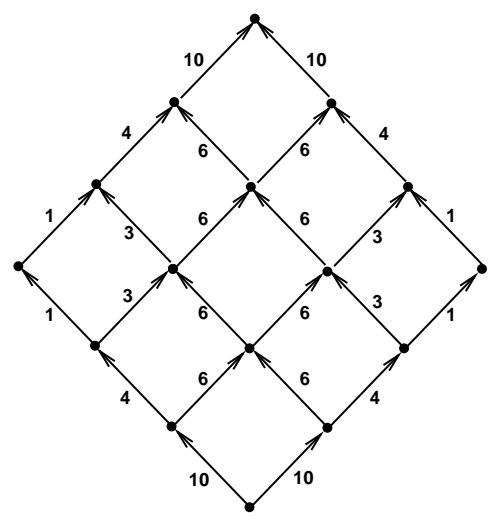

Figure 1: Initial State of Generator

The number on an edge is the number of paths through that edge, which is the product of two binomial coefficients. (There is an easy way to see this. Suppose the source of the graph is $s$ and its sink is $t$. Consider some edge, say $a \rightarrow b$. A path through this edge must go from $s$ to $a$, and from $b$ to $t$, though the sublattices determined by these vertices.)

To generate a string, we use the edge numbers out of each successive vertex (suitably weighted) as probabilities. The number on each edge taken is then decremented by 1 . For example, if we associate the characters 0 and 1 with right and left branches in the graph, then after generating 011001, we would have the state given by Figure 2.

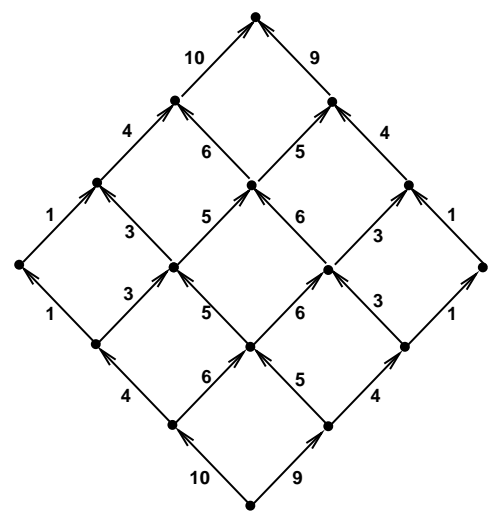

Figure 2: After Generating 011001

We observe that at all times, the probability of taking a given edge is proportional to the number on that edge. (This is easily verified by induction, using the invariant that for every vertex, the edges 
pointing into it have the same sum as the edges pointing out.) For example, consider the edge labelled 6 in the fourth row from the bottom, above. By considering all the ways to get to the preceding vertex, this probability is

$$
\left(\frac{10}{19} \cdot \frac{6}{10} \cdot \frac{6}{11}+\frac{9}{19} \cdot \frac{5}{9} \cdot \frac{6}{11}+\frac{9}{19} \cdot \frac{4}{9} \cdot \frac{3}{4}\right) \frac{6}{9}=\frac{6}{19} .
$$

This allows us to associate a martingale with each edge of the graph. For a given edge, let $X_{i}$ be its number after the $i$-th trial. Then we have

$$
E\left[X_{i+1} \mid X_{i}\right]=X_{i}-\operatorname{Pr}[\text { edge is taken }]=X_{i}-\frac{X_{i}}{\text { total for edges at same level }}=X_{i}\left(1-\frac{1}{N-i}\right) .
$$

As before, we conclude that $Z_{i}=\frac{N}{N-i} X_{i}$ is a martingale. Consequently,

$$
E\left[X_{i}\right]=\frac{N-i}{N} X_{0}
$$

Our goal is now to show that $O\left(n^{3}\right)$ redundancy is sufficient to obtain exponentially small error. This is proved in Theorem 7. A refinement of this argument then reduces the redundancy further to $O(n)$, in Theorem 4. Our principal tool for this will be a generalization of martingale theory to finite-dimensional Euclidean spaces.

Let $\mathbf{R}^{r}$ denote $r$-dimensional Euclidean space, with the norm $\|x\|_{2}=\left(\sum x_{i}^{2}\right)^{1 / 2}$. (We will drop the subscript unless other norms are called into play.) A sequence $W_{0}, W_{1}, \ldots$ of $\mathbf{R}^{r}$-valued random variables is a martingale if $E\left[\left\|W_{i}\right\|\right]<\infty$ and

$$
E\left[W_{i} \mid W_{i-1}\right]=W_{i-1}
$$

Its difference sequence is $d_{i}=W_{i}-W_{i-1}$.

Theorem 5 can be generalized to $r$-dimensional space as well.

Theorem 6 (Hoeffding/Azuma in $r$-space) Let $\left\{W_{i}\right\}_{i=0}^{\infty}$ be a martingale in $\mathbf{R}^{r}$, with difference sequence $\left\{d_{i}\right\}$. Let $\sum_{i=1}^{t}\left\|d_{i}\right\|^{2} \leq D$. Then

$$
\operatorname{Pr}\left[\exists i \leq t\left\|W_{i}-W_{0}\right\|>\lambda\right] \leq 2 \exp \left(-\frac{\lambda^{2}}{2 D}\right) .
$$

Proof: $\quad$ Take $f_{i}=\left(W_{i}-W_{0}\right) / \sqrt{D}$ and $p=2$ in Theorem 3 of Pinelis [13].

We now prove a redundancy bound of $O\left(n^{3}\right)$ for the $\left(\begin{array}{c}n \\ n / k\end{array}\right)$-generator, since this is simpler than proving a bound of $O(n)$. In Theorem 4, we then improve the redundancy bound to $O(n)$, by modifying the proof of Theorem 7 .

Theorem 7 Let $k>1$ be an integer. A redundancy of $R=n^{3}$ is sufficient for the $\left(\begin{array}{c}n \\ n / k\end{array}\right)$-generator, to obtain exponentially small error. More precisely, suppose that $N(=R M) \geq 8 c M \log M, n \geq 2$, and the redundancy satisfies $R \geq n^{3}$. Then all $M=\left(\begin{array}{c}n \\ n / k\end{array}\right)$ strings are generated with probability at least $1-2^{-\Omega(n)}-M^{1-c}$.

Proof: Let $X_{1}, \ldots, X_{r}$ be the edge numbers at some level, whose initial values are $q_{1} N, \ldots, q_{r} N$, with $\sum q_{j}=1$. (In urn language, $X_{j}$ is the number of red balls in some urn.) Then

$$
W_{i}=\frac{N}{N-i}\left(\frac{X_{1}}{q_{1} N}, \ldots, \frac{X_{r}}{q_{r} N}\right)
$$


is a martingale. At each time step exactly one of edge numbers, say $X_{j}$, will decrease by 1 . Using the bound $X_{j} /\left(q_{j} N\right) \leq 1$, we see that

$$
\left\|d_{i}\right\|^{2} \leq(r-1)(\text { change in } N /(N-i))^{2}+\left(q_{j} N\right)^{-2}\left(\text { change in } N X_{j} /(N-i)\right)^{2} .
$$

Since $i \leq N / 2$,

$$
\text { change in } \frac{N}{N-i}=O(1 / N)
$$

and (as in Lemma 3)

$$
\text { change in } \frac{N X_{j}}{N-i}=O(1) \text {. }
$$

Therefore,

$$
\left\|d_{i}\right\|^{2}=O\left(r / N^{2}\right)+O\left(1 /\left(q_{j} N\right)^{2}\right)
$$

if $X_{j}$ changes at step $i$.

Summing over all $i \leq N / 2$, and observing that $r \leq n$, we get

$$
\sum_{i=1}^{N / 2}\left\|d_{i}\right\|^{2}=O(n / N)+O\left(\sum_{j} \frac{\Delta_{j}}{\left(q_{j} N\right)^{2}}\right),
$$

where $\Delta_{j}$ is the number of times that $X_{j}$ changes. Let $R$ be the redundancy, and let $M$ be the number of distinct strings to be generated. For our graphs, we will have $n \leq M$, so

$$
\frac{n}{N}=\frac{n}{M R}=O(1 / R)
$$

Also, $\Delta_{j} \leq q_{j} N$, since $X_{j}$ cannot decrease below 0 . So

$$
\sum_{j} \frac{\Delta_{j}}{\left(q_{j} N\right)^{2}} \leq \sum_{j} \frac{1}{q_{j} N}=\frac{1}{R} \sum_{j} \frac{1}{q_{j} M}=O(1 / R) .
$$

Here we have observed that $q_{j} M$ is a product of binomial cofficients, and used the estimate $\sum_{i=0}^{m}\left(\begin{array}{c}m \\ i\end{array}\right)^{-1}=$ $O(1)$. (See, e.g., Sury [15].) Putting these together we see that

$$
D=\sum_{i=1}^{N / 2}\left\|d_{i}\right\|^{2} \leq C / R
$$

for some $C>0$.

Let $\|x\|_{\infty}=\max \left\{\left|x_{j}\right|\right\}$. Evidently, we have

$$
\|W\|_{\infty} \leq\|W\|_{2}
$$

so by Theorem 6 we have

$$
\begin{aligned}
\operatorname{Pr}\left[\exists i \leq N / 2\left\|W_{i}-W_{0}\right\|_{\infty}>\lambda\right] & \leq \operatorname{Pr}\left[\exists i \leq N / 2\left\|W_{i}-W_{0}\right\|_{2}>\lambda\right] \\
& \leq 2 \exp \left(-R \lambda^{2} /(2 C)\right) .
\end{aligned}
$$


Consider some edge number $X_{j}$. Define $F_{j}$ to be

$$
F_{j}=\frac{X_{j}}{E\left[X_{j}\right]}
$$

and call this edge good if

$$
\left|F_{j}-1\right| \leq \frac{1}{2 n}
$$

We note that $F_{j}$ is a martingale with mean 1 , indeed, it equals the $j$-th component of $W$. By $\left(^{*}\right)$, the probability that all edge numbers are good for all times $\leq N / 2$ is at least

$$
1-2 n \exp \left(-R /\left(A n^{2}\right)\right)
$$

for some $A>0$.

Consider some string $s$, associated with the path $e_{1}, \ldots, e_{n}$ in the graph. Let $X_{i}$ be the edge number for $e_{i}$, and let $Y_{i}$ be the sum of the numbers on the two edges preceding $e_{i}$. (For $i=1$ we can take $Y_{1}=N-i$.) Then we have

$$
\operatorname{Pr}[s]=\frac{X_{1}}{Y_{1}} \cdots \frac{X_{n}}{Y_{n}}=\frac{X_{1}}{E\left[X_{1}\right]} \frac{E\left[Y_{1}\right]}{Y_{1}} \cdots \frac{X_{n}}{E\left[X_{n}\right]} \frac{E\left[Y_{n}\right]}{Y_{n}} \frac{1}{M},
$$

since $E\left[X_{i}\right] / E\left[Y_{i}\right]$ equals the initial value of $X_{i} / Y_{i}$. If all edge numbers are good, then we have

$$
\operatorname{Pr}[s] \geq \frac{(1-1 /(2 n))^{n}}{(1+1 /(2 n))^{n}} \cdot \frac{1}{M} \geq(1-1 / n)^{n} \frac{1}{M} \geq \frac{1}{4 M},
$$

provided that $n \geq 2$. (To handle the $Y_{i}$ 's we will need the following lemma: Let $x / y$ and $z / w$ be within $\epsilon$ of 1 , then the same is true for $(x+z) /(y+w)$. We are also using the inequality $(1-x) /(1+x) \geq 1-2 x$.

As in the analysis of the diamond graphs, we consider another urn model $U^{\prime}$. This acts like our generator until some edge number (= number of red balls) goes bad, at which point the urn for that edge is refilled. If $E_{i}(s)$ denotes the event that the string $s$ is not generated in the first $i$ trials, then we have (as in the analysis for the diamond graphs)

$$
\operatorname{Pr}\left[\exists s E_{N / 2}(s)\right] \leq M\left(1-\frac{1}{4 M}\right)^{N / 2}+\operatorname{Pr}[B],
$$

where $B$ denotes the event that some edge number goes bad during one of the first $N / 2$ trials. By our analysis

$$
\operatorname{Pr}[B] \leq 2 n \exp \left(-R /\left(A n^{2}\right)\right),
$$

which is $2^{-\Omega(n)}$ if we choose $R=n^{3}$. (Comment: we can reduce $R$ somewhat at the price of making this estimate larger.) Choosing $N=8 c M \log M$, we get

$$
M\left(1-\frac{1}{4 M}\right)^{N / 2} \leq M^{1-c}
$$

These estimates easily imply the theorem.

To reduce the redundancy to $O(n)$, we take advantage of the fact that $\left(\begin{array}{c}n \\ n / k\end{array}\right)$-generators have few edges with "small" edge numbers. Define an edge number to be small if it is at most $\left(\begin{array}{c}n / k+4 \\ 4\end{array}\right)=\Theta\left(n^{4}\right)$, assuming that $k$ is a constant independent of $n$. 
Lemma 4 For fixed $k$, the number of small edges in the $\left(\begin{array}{c}n \\ n / k\end{array}\right)$-generator is $O(1)$.

Proof: We already noted that an edge number is the product of two binomial coefficients; in fact, the possible edge numbers are

$$
\left(\begin{array}{c}
i-1 \\
j
\end{array}\right)\left(\begin{array}{c}
n-i \\
n / k-j
\end{array}\right), 1 \leq i \leq n, 0 \leq j \leq n / k, j \leq i-1 \text { and } n / k-j \leq n-i
$$

or

$$
\left(\begin{array}{c}
i-1 \\
j-1
\end{array}\right)\left(\begin{array}{c}
n-i \\
n / k-j
\end{array}\right), 1 \leq i \leq n, 1 \leq j \leq n / k, j-1 \leq i-1 \text { and } n / k-j \leq n-i .
$$

For example, suppose that in the example of Figure 2, rows of nodes are numbered $i=0,1, \ldots, 6$ from bottom to top and within each row, nodes are numbered $j=0,1, \ldots$. Then the edge numbers of the edge(s) into the $j$ th node in row $i$ are given by the above expressions.

In what follows, assume that $k>2$; the case when $k=2$ is similar. We claim that for sufficiently large $n$, if an edge number is small, then one of the following conditions must hold:

(i) $n / k-4<i \leq n / k+4$ and $j>n / k-4$, or

(ii) $n-n / k-4<i \leq n-n / k+4$ and $j \leq 4$.

Clearly there are $O(1)$ pairs $(i, j)$ satisfying these conditions.

To prove our claim, we consider all the possible cases for $i$ and $j$ that do not meet the conditions, and show in every case that the binomial product is not small.

Case a: $i \leq n / k-4$. In this case, $n-i \geq n-n / k+4 \geq n / k+4$ (since we are assuming that $k>2$ ). Also, since $j \leq i$ we have $4 \leq n / k-j \leq n / k$. Hence,

$$
\left(\begin{array}{c}
n-i \\
n / k-j
\end{array}\right) \geq\left(\begin{array}{c}
n-n / k+4 \\
4
\end{array}\right) \geq\left(\begin{array}{c}
n / k+4 \\
4
\end{array}\right)
$$

Case b: $n / k-4<i \leq n / k+4$ and $j \leq n / k-4$. In this case, $n / k-j \geq 4$. Also, for sufficiently large $n, n-i \geq n-n / k-4 \geq n / k+4$. (Again we use the fact that $k>2$.) Hence,

$$
\left(\begin{array}{c}
n-i \\
n / k-j
\end{array}\right) \geq\left(\begin{array}{c}
n / k+4 \\
n / k-j
\end{array}\right) \geq\left(\begin{array}{c}
n / k+4 \\
4
\end{array}\right) .
$$

Case c: $n / k+4<i \leq n-n / k-4$. In this case, for sufficiently large $n$, either $n / k-j \geq 4$ or $j-1 \geq 4$. If $n / k-j \geq 4$ then

$$
\left(\begin{array}{c}
n-i \\
n / k-j
\end{array}\right) \geq\left(\begin{array}{c}
n / k+4 \\
n / k-j
\end{array}\right) \geq\left(\begin{array}{c}
n / k+4 \\
4
\end{array}\right)
$$

If $j-1 \geq 4$ then

$$
\left(\begin{array}{c}
i-1 \\
j
\end{array}\right) \geq\left(\begin{array}{c}
n / k+4 \\
4
\end{array}\right)
$$

The same is true of $\left(\begin{array}{c}i-1 \\ j-1\end{array}\right)$.

Case d: $n-n / k-4<i \leq n-n / k-4$ and $j>4$. Again we use the fact that for sufficiently large $n$, $n-n / k-4 \geq n / k+4$. In this case,

$$
\left(\begin{array}{c}
i-1 \\
j
\end{array}\right) \geq\left(\begin{array}{c}
n-n / k-4 \\
j
\end{array}\right) \geq\left(\begin{array}{c}
n / k+4 \\
j
\end{array}\right) \geq\left(\begin{array}{c}
n / k+4 \\
4
\end{array}\right) .
$$

The same is true of $\left(\begin{array}{c}i-1 \\ j-1\end{array}\right)$. 
Case e: $i>n-n / k+4$. In this case, since $n / k-j \leq n-i$, we have that $n / k-j<n / k-4$ and so $j>4$. Therefore,

$$
\left(\begin{array}{c}
i-1 \\
j
\end{array}\right) \geq\left(\begin{array}{c}
n-n / k+4 \\
4
\end{array}\right) \geq\left(\begin{array}{c}
n / k+4 \\
4
\end{array}\right)
$$

The same is true of $\left(\begin{array}{c}i-1 \\ j-1\end{array}\right)$.

Theorem 4 Let $k>1$ be an integer. A redundancy of $R=\Theta(n)$ is sufficient for the $\left(\begin{array}{c}n \\ n / k\end{array}\right)$-generator, to obtain exponentially small error. More precisely, suppose that $N(=R M) \geq 8 c M \log M$. Then for sufficiently large $n$, all $M=\left(\begin{array}{c}n \\ n / k\end{array}\right)$ strings are generated with probability at least $1-2^{-\Omega(n)}-M^{1-c}$.

Proof: To improve the redundancy bound of Theorem 7, we reweight the components of the martingale $W_{i}$ containing small edge numbers. Let

$$
w_{i}= \begin{cases}1, & \text { if } X_{i} \text { is not small } \\ n, & \text { if } X_{i} \text { is small }\end{cases}
$$

Then

$$
W_{i}=\frac{N}{N-i}\left(\frac{X_{1}}{w_{1} q_{1} N}, \ldots, \frac{X_{r}}{w_{r} q_{r} N}\right)
$$

is a martingale. A similar derivation as before shows that for this martingale,

$$
\sum_{i=1}^{N / 2}\left\|d_{i}\right\|^{2}=O(n / N)+O\left(\sum_{j \mid X_{j} \text { is not small }} \frac{1}{q_{j} N}\right)+O\left(\sum_{j \mid X_{j} \text { is small }} \frac{1}{n^{2} q_{j} N}\right) .
$$

From Lemma 4, the number of small edge numbers at any level is $O(1)$. Since $q_{j} N \geq R=\Theta(n)$,

$$
\sum_{j \mid X_{j} \text { is small }} \frac{1}{n^{2} q_{j} N}=O\left(1 / n^{3}\right) .
$$

Also, since the edge numbers that are not small are $\Omega\left(n^{4}\right)$,

$$
\sum_{j \mid X_{j} \text { is not small }} \frac{1}{q_{j} N}=O\left(1 / n^{3}\right) .
$$

Finally, since $\left(\begin{array}{c}n \\ n / k\end{array}\right)=\Omega\left(n^{3}\right)$,

$$
n / N=O\left(1 / n^{3}\right)
$$

Hence, we have that

$$
D=\sum_{i=1}^{N / 2}\left\|d_{i}\right\|^{2} \leq C / n^{3}
$$

for some $C>0$. By Theorem 6 , we now have

$$
\begin{aligned}
\operatorname{Pr}\left[\exists i \leq N / 2\left\|W_{i}-W_{0}\right\|_{\infty}>\lambda\right] & \leq \operatorname{Pr}\left[\exists i \leq N / 2\left\|W_{i}-W_{0}\right\|_{2}>\lambda\right] \\
& \leq 2 \exp \left(-n^{3} \lambda^{2} /(2 C)\right) .
\end{aligned}
$$


Now let

$$
F_{j}=\frac{X_{j}}{w_{j} E\left[X_{j}\right]}
$$

Call $X_{j}$ good if

$$
\left|F_{j}-\frac{1}{w_{j}}\right| \leq \frac{1}{l n}
$$

where $l$ satisfies $(1-2 /(\ln ))^{n}\left(\frac{l-1}{l+1}\right)^{S} \geq 1 / 4$ for all $n$. Here, $S$ is the constant of Lemma 4, i.e. an upper bound on the number of small edges of a $\left(\begin{array}{c}n \\ n / k\end{array}\right)$-generator. By $(* *)$, the probability that all edges are good for all times $\leq N / 2$ is at least

$$
1-2 n \exp (-A n)
$$

for some constant $A>0$.

Let $s$ be some string associated with a path in the graph. By Lemma 4, this path has at most $S$ small edge numbers. Hence, calculating as in Theorem 7,

$$
\operatorname{Pr}[s] \geq \frac{(1-1 /(\ln ))^{n}}{(1+1 /(\ln ))^{n}} \frac{(1-1 / l)^{S}}{(1+1 / l)^{S}} \cdot \frac{1}{M} \geq\left(1-\frac{2}{l n}\right)^{n}\left(\frac{l-1}{l+1}\right)^{S} \frac{1}{M} \geq \frac{1}{4 M},
$$

by our choice of $l$. Hence,

$$
\operatorname{Pr}\left[\exists s E_{N / 2}(s)\right] \leq M\left(1-\frac{1}{4 M}\right)^{N / 2}+\operatorname{Pr}[B],
$$

where $\operatorname{Pr}[B] \leq 2 n \exp (-A n)$. Choosing $N \geq 8 c M \log M$, we get

$$
M\left(1-\frac{1}{4 M}\right)^{N / 2} \leq M^{1-c}
$$

and the analysis is completed as in Theorem 7.

\section{Conclusions}

In this paper, we have described algorithms for 3-Coloring and Independent Set that use only the restricted set of operations proposed by Adleman, while using a solution space of only $1.89^{n}$ and $1.51^{n}$, respectively. These algorithms represent a big improvement over naive exhaustive search. If we again assume Adleman's speculation that a solution space of size $2^{70}$ is feasible, we can solve instances of 3-Coloring and Independent Set with 76 and 118 vertices respectively, in contrast with the limits of 44 and 70, respectively, for the naive algorithms.

These improvements are not sufficient to claim that DNA computation is practical for such problems. However, there is no reason to assume that they are optimal. Improved algorithms may be possible on our model, or if new operations are added to the model. We note that heuristic algorithms may also be useful in the DNA computing model.

Finally, we present the first analysis of errors in the solution space generation phase due to splitting of the contents of test tubes. This is a very basic operation, so our analysis is likely to be useful in any potential application of DNA computation. We show how to generate the set of binary strings of length $n$ with exactly $k$ 1's, using $O(n k)$ operations and a redundancy of $\Theta(n)$. An alternative 
method of generating this set of strings was proposed by Dan Boneh (personal communication), using an additional operation that separates strings according to their length (see [5] for a description of this operation).

We thank the anonymous referee for several helpful comments that improved the presentation of this paper.

\section{References}

[1] L. M. Adleman, Molecular Computation of Solutions to Combinatorial Problems, Science 266, 11 November 1994, pp. 1021-1024.

[2] L. M. Adleman, On Constructing a Molecular Computer, Manuscript, Department of Computer Science, University of Southern California, 1995.

[3] M. Amos, A. Gibbons and D. Hodgson, Error-resistant Implementation of DNA Computations, Research Report CS-RR-298, Department of Computer Science, University of Warwick, Coventry, UK. January 1996.

[4] R. Beigel and D. Eppstein, 3-Coloring in Time $O\left(1.3446^{n}\right)$ : a No-MIS Algorithm, Proc. 36th Symposium on Foundations of Computer Science, IEEE Computer Society Press, Los Alamitos, 1995, pp. 444-453.

[5] D. Boneh, D. Dunworth, R.J. Lipton and J. Sgall, On the Computational Power of DNA, Technical Report Number TR-499-95, Computer Science Department, Princeton University, Princeton, New Jersey, 1995.

[6] S. P. Fodor, J. L. Read, M. C. Pirrung, L. Stryer, A. T. Lu and D. Solas, Light-Directed, Spatially Addressable Parallel Chemical Synthesis, Science 251, 1991, pp. 767-773.

[7] D. E. Knuth, Mariages Stables et Leurs Relations avec d'autres Problèmes Combinatoires, Presses Univ. Montreal, 1976.

[8] G.R. Grimmett and D.R. Stirzaker, Probability and Random Processes (second edition), Oxford University Press, 1992.

[9] F. Harary, Graph Theory, Addison-Wesley, Reading, MA.

[10] R.J. Lipton, DNA Solution of Hard Combinatorial Problems, Science 268, 28 April 1995, pp. 542548.

[11] R. Motwani and P. Raghavan, Randomized Algorithms, Cambridge University Press, 1995.

[12] A. C. Pease, D. Solas, E. J. Sullivan, M. T. Cronin, C. P. Holmes and S. P. Fodor, Light-Directed Oligonucleotide Arrays For Rapid DNA Sequence Analysis, Proc. Natl. Acad. Sci. USA, 91, May 1994, pp. 5022-5026.

[13] I. Pinelis, An Approach to Inequalities for the Distributions of Infinite-dimensional Martingales, in R. M. Dudley, M. G. Hahn, and J. Kuelbs, ed., Probability in Banach Spaces 8, pp. 128-134. Birkhauser, 1992. 
[14] I. Schiermeyer, Solving 3-Satisfiability In Less Than $1.579^{n}$ Steps, Proc. 6th Workshop on Computer Science Logic, Springer-Verlag, 1993, pp. 379-394.

[15] B. Sury, Sum of the Reciprocals of the Binomial Coefficients, Europ. J. Combinatorics 14, 1993, pp. 351-353.

[16] D. Williams, Probability with Martingales, Cambridge Univ. Press, 1991. 

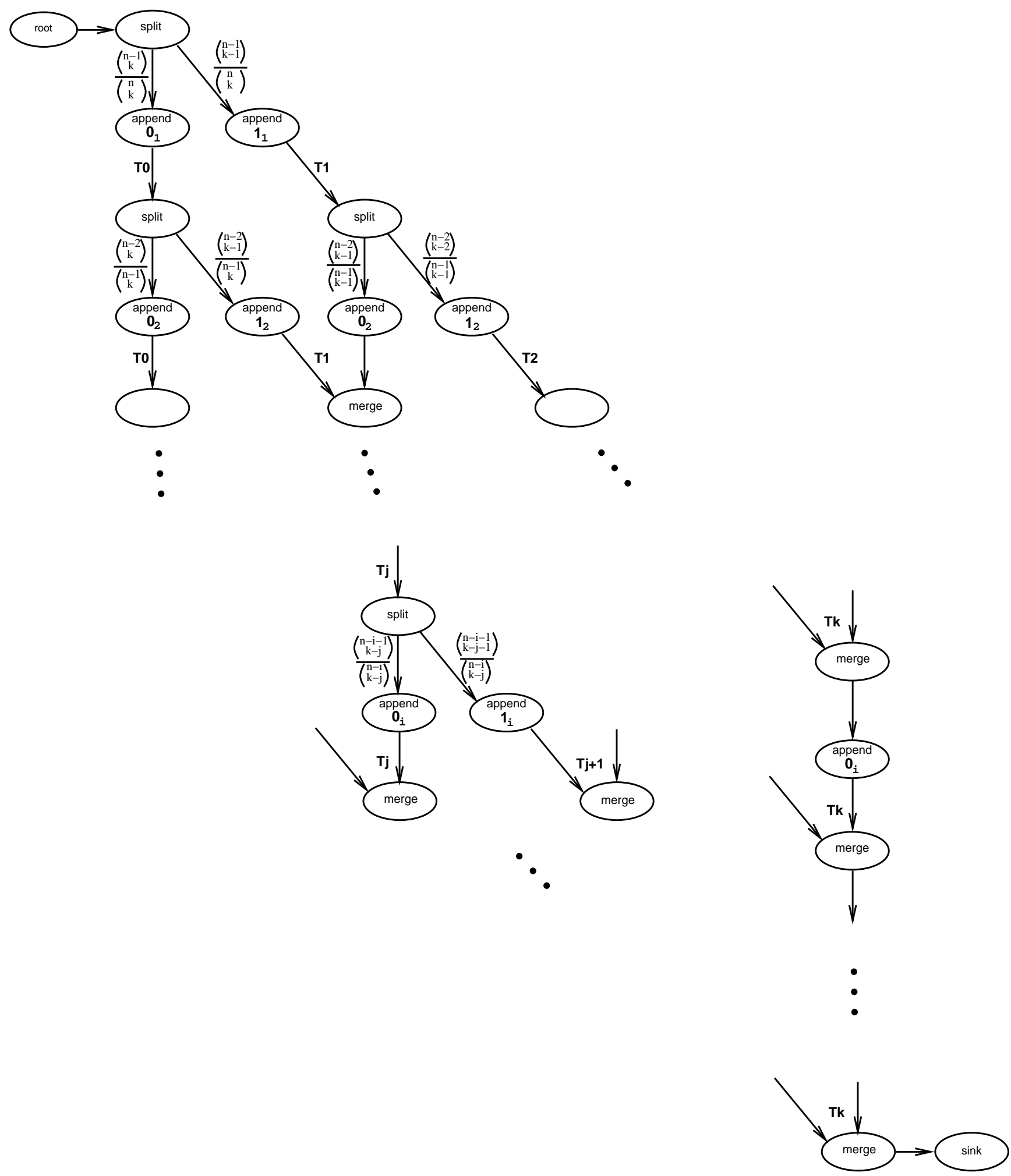

Figure 3: An $n$ choose $k$ generator. The test tube labeling the edge from the root contains all empty strings, and the test tube labeling the edge to the sink contains the desired solution space. Each test tube $T_{i}, 1 \leq i \leq k$ stores strings with $i$ 1's. Initially, $T_{0}$ consists of $n$ choose $k$ empty strings. At the $i$ th stage, for each $j, 1 \leq j<k$, a weighted split is performed on $T_{j}$, so that $\frac{k-j}{n-i}$ of $T_{i}$ 's elements are in one tube and $\frac{n-i-(k-j)}{n-i}$ in the other. We append $1_{i+1}$ to the strings in the first tube and merge it with $T_{j+1}$. To the second tube we append $0_{i+1}$; and merge it with $T_{j} . T_{1}$ and $T_{k}$ are handled somewhat differently than the general case, as shown. 